Estefany Robles

DOI $10.35381 / \mathrm{cm} . v 5 i 9.243$

\title{
Responsabilidad Empresarial Endógena: Una perspectiva para Calidad Empresarial
}

\section{Endogenous Corporate Responsibility: A perspective for Business Quality}

\author{
Estefany Robles \\ estefanyrobleses@hotmail.com \\ Institución Entidad Micro crediticia Bancamia \\ Colombia \\ https://orcid.org/0000-0003-2870-8062
}

Recibido: 23 de abril de 2019

Aprobado: 1 de junio del 2019

\begin{abstract}
RESUMEN
La finalidad de esta investigación fue estudiar la Responsabilidad Empresarial Endógena desde una perspectiva para la calidad Empresarial; Se desarrolló desde el enfoque cualitativo bajo el método fenomenológico. En ese sentido la responsabilidad social interna constituye un nuevo esquema gerencial por medio del cual se alimenta y se ilumina el compromiso del individuo con respecto a la organización. Este artículo en la primera sección hace un recorrido por los conceptos; en la segunda hace una aproximación a las normas y guías; y en la tercera, concluye planteando la importancia que tiene la RSE como parte del éxito de las organizaciones. Concluyendo así que la misma siempre debe ir en busca de la excelencia para estar a la par de los mercados competitivos, pero para lograrlo es necesario el compromiso de todos los miembros que componen una organización independientemente del nivel jerárquico que le corresponda desempeñar.
\end{abstract}

Descriptores: Responsabilidad empresarial; Endógena; Calidad; Desarrollo; Social.

\begin{abstract}
The purpose of this research was to study Endogenous Corporate Responsibility from a perspective for Business quality; It was developed from the qualitative approach under the phenomenological method. In that sense, internal social responsibility constitutes a new management scheme through which the individual's commitment to the organization is nourished and illuminated. This article in the first section takes a tour of the concepts; in the second it makes an approximation to the norms and guides; and in the third, he concludes by raising the importance of CSR as part of the success of organizations. Thus concluding that it must always go in search of excellence to be on a par with competitive markets, but to achieve this it is necessary the commitment of all the members that make up an organization regardless of the hierarchical level that corresponds to perform.
\end{abstract}




\section{Estefany Robles}

Descriptors: Corporate responsibility; Endogenous; Quality; Developing; Social.

\section{INTRODUCCIÓN}

A nivel mundial la economía viene generando aperturas en los mercados internacionales, aunado al fenómeno de la globalización, cambios culturales haciendo que estos aspectos incidan de manera notable en las empresas y el entorno social. Aunado a ello la presión política, el deterioro ambiental, la debilidad institucional e imbatibilidad de la pobreza. Estos factores por considerarse de interés ante la sociedad en general impactan de manera notable la transparencia haciendo que los individuos que la conforman den un vuelco y busquen poner de manifiesto su comportamiento ético a dicha situación.

En este sentido, en las últimas décadas se ha propiciado a nivel mundial específicamente en Latinoamérica, un gran interés por parte de los diferentes sectores, público y privado, en gestionar con visión de responsabilidad social empresarial, en ese sentido Capriotti (2010), apela una nueva expresión, considerando la definición de la responsabilidad social empresarial donde se admiten varias acepciones, pero todas coinciden que se trata de un enfoque basado en un conjunto integral de políticas, prácticas y programas centrados en el respeto por la ética, las personas, las comunidades y el medio ambiente.

Es por ello que la responsabilidad social empresarial ha evolucionado haciendo partícipe a las empresas en la definición del tipo de sociedad a la que aspira tener. Estos cambios van centrados en que las empresas actúen como líderes en cuanto a la solución de problemas, generando iniciativas propias o propuestas que coadyuven con soluciones a las diferentes problemáticas detectadas. Es entonces cuando se hace necesario un cambio de valores en la ciudadanía para poder generar en ellos nuevos roles y dinámicas empresariales, Arias \& Sarmiento (2016).

Analizando entonces el panorama expuesto, la empresa se ha visto obligada a emprender acciones para revisar rutinas o valores, implantando y activando mecanismos complementarios que acceden a regular el funcionamiento e interacción con el entorno, ello con la finalidad de rescatar la confianza social no solo a las comunidades sino también a las personas que realizan trabajos dentro de las empresas, con el objeto de responder de manera efectiva a las diferentes 


\section{Estefany Robles}

normativas jurídicas y extra jurídicas, trascendiendo los dispositivos permitidos o prohibidos en aras de poder incrementar la rentabilidad, entre los cuales se destacan: inversión en el capital humano, mejor relación con los interlocutores, respeto al medio ambiente, entre otros aspectos relevantes.

Es así entonces, como se da origen a la responsabilidad social interna como fenómeno, la cual contempla aspectos como: trabajo en equipo asociados, familia, trabajadores, accionistas, entre otros; es importante señalar que la responsabilidad social interna se hace efectiva una organización desarrolla una toma de decisiones compleja y holística de sí misma, en la búsqueda de un desarrollo de su entorno.

Por ende es importante señalar que en Latinoamérica, la gerencia del siglo XXI esgrime políticas gestionando un nuevo enfoque de responsabilidad social interna, en el que las organizaciones centran su atención o tienen como filosofía el crecimiento de su capital humano, mediante el desarrollo tanto personal como profesional, ofreciendo lugares de trabajos seguros y saludables, gestiones efectivas antes los cambios del mercado, así como también la posibilidad al acceso de la información permitiendo mejorar sus condiciones tanto físicas como contractuales.

Además de ello se han venido incorporando dentro de las políticas empresariales el fomento de la responsabilidad social interna dentro de su gestión, en la cual se incluyan elementos como la satisfacción de los trabajadores, protección del medio ambiente, el bienestar de la organización, las mejoras de las relaciones con el entorno que las rodea, mejorando los resultados económicos como impulsador de actividades que apalanquen el compromiso y el sentido de pertinencia de los trabajadores con la organización.

En ese sentido es importante señalar según el informe emitido por el grupo Gerdau (2013), que en las organizaciones donde la responsabilidad social interna se encuentra implementada como política de gestión dentro de su filosofía organizacional, ésta se convierte en un elemento diferenciador apalancando el posicionamiento y la competitividad de esta en el tiempo, de igual forma, esta le brinda una ventaja competitiva en relación a organizaciones del mismo ramo, a través de esta se pueden ver cristalizados los objetivos de forma eficaz y eficiente, lo cual permite cumplir con expectativas de calidad generadas por el cliente Aldana \& Piña (2017). 


\section{Estefany Robles}

En el mismo orden de ideas, el Libro Verde (2011) plantea las prácticas empresariales responsables en lo social que afectan en primer lugar a los trabajadores, estas se refieren a cuestiones como la inversión en recursos humanos, la salud y la seguridad, así como a la gestión del cambio.

Según lo expuesto, la responsabilidad social interna busca fomentar un buen entorno laboral donde el empleado se sienta involucrado, comprometido con los objetivos de la empresa, facilitando la cohesión interna, para así estimular la productividad y motivación del trabajador, además es una cuestión de coherencia y credibilidad para las empresas que pretendan ser catalogadas como socialmente responsables.

\section{DESARROLLO TEÓRICO}

\section{Responsabilidad Social Empresarial}

La RSE es un nuevo paradigma de la gestión en las empresas, que ayuda al fortalecimiento de la responsabilidad de la empresa en la sociedad. Ejemplo en cuestiones sociales y ambientales (Vergara-Arrieta, \& Carbal-Herrera, 2014). Una definición general de RSE la expresan McWilliams y Siegel (2011), cuando expresan que son "las acciones que aparecen para promover algún bien social, más allá de los intereses de la empresa y lo que es requerido por la ley". Es por ello entonces que puede decirse que no todas las actividades sociales que realice la empresa forman parte de la RSE, sino solo las que vayan más allá de los intereses de la empresa, en este caso de sus actividades de valor. Así mismo, el respaldo por parte del estado en fomentar en las empresas acciones que sean socialmente responsables, es importante puesto que estimula, a partir de beneficios, sobre todo fiscales, a las empresas que realicen este tipo de acciones (Martínez- Bernal, 2013).

Al respecto, la Comisión de las Comunidades Europeas, CCE, (2011), con la publicación del "Libro Verde", define la RSE cómo: "un concepto con arreglo al cual las empresas deciden voluntariamente contribuir al logro de una sociedad mejor y un medio ambiente más limpio". En esta misma línea, Carneiro- Caneda (2013), define a la RSE cómo: "la integración voluntaria, por parte de las empresas de las preocupaciones sociales y morales en sus operaciones comerciales y en las relaciones con sus interlocutores". En relación con estas definiciones, se destaca que la decisión de las organizaciones en ser más responsable socialmente es 


\section{Estefany Robles}

netamente voluntaria, y eso dependerá de qué tanto esta sea más o menos responsable.

En relación a lo expuesto puede decirse que la Responsabilidad Social se clasifica en dos dimensiones: Externa que es cuando la empresa u organización se relaciona con el entorno y la interna como su nombre lo indica se refiere a la empresa en sí misma.

\section{Dimensión interna}

En lo relacionado a esta dimensión, Marchart (2006) direcciona la responsabilidad social empresarial interna al entorno de la organización, al mismo tiempo considera como grupos de interés a los directivos y empleados, involucrando en sus políticas de gestión el escenario en el que se desarrollan las actividades de la empresa, en el proceso productivo o en la toma de decisiones. Asimismo, el autor citado expone que dentro de esta dimensión se incluye la integración de la responsabilidad en la empresa como su estrategia de comunicación corporativa, identificando los objetivos, o metas necesarias para alcanzar proporcionando el compromiso de todos los actores empresariales

Por su parte, Guibert (2009) plantea en las empresas practicas responsables en lo social las cuales afectan en primer lugar a los trabajadores refiriéndose a cuestiones como la inversión en recursos humanos, salud, seguridad, gestación del cambio, considerando que las practicas respetuosas con el medio ambiente se fundamentan con la gestión de los recursos naturales utilizados en la producción, este tipo de responsabilidad puede recogerse en dos aéreas y estas son; todo aquello afectando al bienestar y calidad de vida de los trabajadores, además con las condiciones medioambientales en las que tienen lugar los procesos de producción empresariales. Por su parte, Reyno (2007) considera los empleados, dueños y accionistas, para los cuales la responsabilidad social va a actuar proporcionando una calidad de vida, un entorno seguro, más atractivo desarrollando sus funciones entre otros beneficios, así mismo en esta perspectiva las empresas fundamentan su actuación en el compromiso de mantener la coherencia de sus acciones dentro de la organización, entregando a la vez certidumbre y transparencia en sus relaciones, propiciando un ambiente de trabajo favorable.

Sobre el particular, Fernández (2009) relaciona la responsabilidad social empresarial 


\section{Estefany Robles}

con los ámbitos internos de la organización, afirmando que la misma afecta a sus trabajadores, a su impacto medioambiental, a la gestión de las materias primas, a sus condiciones de trabajo o a la peligrosidad de sus productos, según este autor esta responsabilidad está referida a incrementar la participación y el trabajo en equipo en los colaboradores integrantes de la organización.

De la misma manera, Delgado (2012) plantea la responsabilidad social interna como un elemento de cohesión y motivación permitiendo incrementar el compromiso, y en consecuencia la productividad de todas las personas que trabajan en ella, la misma debe manejar objetivos como; el desarrollo y capacitación del personal, la retención del talento, la difusión de la ética corporativa, la reconciliación interna, la mejora del ambiente de trabajo entre otros orientados al bienestar común.

La RSE interna trata sobre el talento humano dentro de la organización, en cómo ser socialmente responsables con los trabajadores. De acuerdo con Moreno y colaboradores (2010): "en la RSE interna la empresa debe incidir con especial atención en crear y fomentar una cultura de empresa, que esté presente de manera real y no fingida en todos sus miembros". Para lograr esta cultura, la empresa debe inculcar $y$ fortalecer los valores en las personas, ya que finalmente son ellas las que hacen o denotan a la organización.

La responsabilidad social y el medio en que se mueve el trabajador deben ir más allá de los solos postulados en las políticas de la empresa; se necesita inversión de recursos económicos, conseguir un equilibrio entre el rol del trabajador y el papel que este representa en la sociedad (García-Rubiano, \& Forero-Aponte, 2015). Las empresas deben ver por encima de los muros y la productividad de sus empleados, deben visualizar los demás factores que pueden afectar el entorno de vida de sus funcionarios.

Así mismo, las empresas pueden encontrar en el fenómeno de la pobreza una oportunidad y convertirla en un negocio lucrativo, tal y como lo señalan Balza-Franco y Cardona-Arbeláez (2015): "Por su lado, las grandes compañías transnacionales que en parte se benefician del fenómeno y también lo alimentan, intentan mitigar los efectos de la pobreza con el desarrollo de diversos programas de RSE o con acciones de filantropía".

La Comisión de las Comunidades Europeas CCE (2011), plantea que la dimensión 


\section{Estefany Robles}

interna de la RSE afecta a los trabajadores en aspectos cómo: la inversión en recursos humanos, la salud y la seguridad, y la gestión del cambio, como se indica a continuación:

La gestión en recursos humanos. El reto que afrontan las empresas en cuanto a los recursos humanos es en la atracción de trabajadores cualificados y en el mantenimiento de las personas en la organización (Parra-Penagos, \& RodríguezFonseca, 2016).

En este aspecto, la RSE debería tener: aprendizaje permanente, responsabilización de los trabajadores, mejora de información en la empresa, equilibro entre trabajo, familia y ocio, mayor diversidad de recursos humanos, igualdad de retribución y de perspectivas profesionales para las mujeres, entre otros. En últimas, la importancia de generar un entorno que estimule el aprendizaje permanente de los trabajadores, en particular de aquellos que tengan un menor nivel educativo, es uno de los mayores retos y apuestas que debe tener toda empresa que se considere en un ambiente de RSE (Comisión de las Comunidades Europeas, 2011).

\section{Dimensión externa}

En la dimensión externa de la RSE, de acuerdo con Carroll (1999), se pueden considerar:

Responsabilidad Social: se concreta en la realización de obras de interés social o cualquier otro tipo de práctica que suponga un beneficio social.

Responsabilidad Económica: la cual afecta a la búsqueda del máximo beneficio, así como al logro del mayor valor posible para el accionista: ello se consigue a través de la mejora de la eficiencia y de la productividad.

Responsabilidad medioambiental: corresponde a la obligación que mantiene cualquier empresa de cuidar y preservar tanto el entorno como la naturaleza en su conjunto.

Señala Becerra-Gualdrón, \& Cruz-Vásquez, (2014) es incongruente que una empresa se fortalezca cada año desde lo económico y su entorno esté deteriorado, sin acciones correctivas ni preventivas que puedan mejorar la problemática. Asimismo, las empresas deben tener en cuenta el entorno físico local, el mantenimiento de un aire puro, aguas no contaminadas o carreteras 


\section{Estefany Robles}

descongestionadas tanto para su producción y oferta de servicios como para la calidad de vida de aquellas comunidades locales que conviven con la empresa (Alvarez-Meneses, 2013).

\section{Elementos de la Responsabilidad Social Empresarial Interna}

Los elementos que caracterizan y componen la responsabilidad social empresarial interna de acuerdo con el Libro Verde (2011) indica que para cualquier organización son mucho más fáciles de planificar y controlar, en cuanto a la dimensión de sus recursos y capacidades, pero siempre controladas e interiorizadas por la organización.

Por su parte, expone Güerere (2008) los elementos que respectan a la responsabilidad social interna están direccionados con los empleados, tomando en cuenta la gestión de los recursos naturales que deben realizarse en el proceso de producción, según lo expresado por la Comunidad Europea, ha de contemplar también la gestión de recursos humanos, salud y seguridad en el lugar de trabajo, adaptación al cambio incluyendo la gestión de impacto ambiental de los recursos naturales.

Del mismo modo, Ventura (2013) manifiesta los elementos de la responsabilidad social interna a través de una visión de gestión del recurso humano intentando incorporar en sus áreas de actuación elementos como la selección, el reclutamiento, salud y seguridad en el trabajo, el desarrollo profesional, incluyendo el control de situaciones especiales. La sensibilización sobre las cuestiones sociales planteadas por la responsabilidad social empresarial hace que el departamento de recursos humanos no pueda aplicar políticas irrespeten los derechos humanos, la legitimidad de las negociaciones colectivas, el fomento de la igualdad de derechos y oportunidades.

Continuando con lo expuesto por el autor citado, la responsabilidad social interna supone que el empresario debe tener en cuenta a la hora de implantar en su empresa una estrategia todos y cada uno de los factores internos y externos, de no ser así la estrategia nunca estará completa, corriendo el riesgo de caer en un saco roto todo el trabajo al momento de darse una mala práctica en aquel campo al que no se le ha prestado la suficiente atención. Los elementos internos están 


\section{Estefany Robles}

compuestos por: gestión de los recursos humanos, seguridad y salud en el trabajo, adaptación al cambio, gestión del impacto ambiental y de los recursos naturales. Además, estos elementos de la responsabilidad social interna radican en la forma de cómo estos impactan y se relaciona con el contexto interno, con la finalidad de continuar evolucionando, considerando la forma en cómo el recurso humano puede ayudar en el vertiginoso ritmo empresarial. De allí la importancia que tienen las empresas en invertir en dos aspectos fundamentales salud y educación para la formación de un capital humano.

\section{Capital Humano}

Guédez (2006) señala el concepto de capital humano procedente de la necesidad de redimensionar el aporte del ser humano a los procesos de productividad empresarial y de favorecer las dinámicas del desarrollo de los países. Mientras que para Ventura (2013) quizás uno de los desafíos más importantes a los que se enfrentan hoy en día las empresas es atraer a trabajadores cualificados y lograr su permanencia.

De igual forma, Bericat y Echavarren (2008) aseguran que el capital humano es un recurso productivo el cual no solo favorece al desarrollo económico, sino que es uno de los apoyos básicos para alcanzar la igualdad de oportunidades en la sociedad, además del correcto funcionamiento y calidad de los sistemas educativos son vitales para la formación del capital humano durante los diferentes períodos educativos.

En palabras de López y Grandio (2010) el capital humano es el conjunto de habilidades, conocimientos y competencias de las personas que trabajan en las empresas, como una fuente incuestionable de ventajas competitivas a largo plazo, es el activo más importante, el cual genera el capital estructural y relacional de la compañía. En referencia a lo expuesto por los autores el capital humano puede considerarse como todas las habilidades y destrezas que tienen los individuos y que pueden poner en practica dentro de una organización para poder generar el capital estructural y relacional haciendo uso de su capacidad creativa e inventiva.

\section{Seguridad y Salud en el Trabajo}

En relación a la salud y seguridad en el trabajo, se hace referencia a la prevención de riesgos laborales, pero no como un mero cumplimiento de preceptos legales, sino 


\section{Estefany Robles}

concebido en el hecho de que la calidad de producción o prestación de servicios incide de manera directa en la calidad del producto o servicio final ofrecido al mercado. Al respecto, Vidal (2003) señala que los indicadores referidos a este ámbito tienen por objeto la prevención junto con el aumento del nivel de la salud y la seguridad en el sitio de trabajo.

De acuerdo con lo indicado por Güerere (2008), la tendencia de las organizaciones a incluir criterios de salud y seguridad en el trabajo en su régimen de contratación ha permitido adoptar regímenes generales basados en requisitos uniformes que deben ser respetados por los programas de formación considerando también los de gestión de la salud y la seguridad de los contratistas, los cuales permiten a terceros realizar la certificación o dar la aprobación inicial del contratista para de esta forma vigilar la mejora continua del programa.

Por su parte, Cortés (2012) define este concepto como una seguridad integrada en los proyectos 0 en el diseño de obras, instalaciones, maquinarias, equipos 0 procesos, ya que las medidas de prevención adoptadas en dichas fases, aparte de ser menos costosas, resultan ser más eficaces que las efectuadas en los procesos de producción ya en funcionamiento.

Para Molto (2013), la seguridad en el trabajo es un conjunto de medidas técnicas, educativas, médicas y psicológicas implementadas para prevenir accidentes, tendientes a eliminar las condiciones inseguras del ambiente y a instruir o convencer a las personas acerca de la necesidad de implementación de prácticas preventivas. Por ello, es importante considerar la salud y seguridad en el lugar de trabajo no solo para los empleados y trabajadores de la empresa, sino para aquellos que forman parte de los contratistas y proveedores.

En el mismo orden de ideas, en cuanto a la salud y seguridad en el lugar de trabajo indica la Comisión de las Comunidades Europeas, (2011) se ha venido fortaleciendo en los últimos años, el garantizar a los trabajadores condiciones óptimas para que puedan ejercer sus funciones dentro de la empresa como característica fundamental, por cuanto afecta su eficiencia y productividad. Señala el organismo citado, que la calidad del proceso de producción repercute de manera directa en la calidad del producto o servicio que ofrece la empresa en el mercado, por lo tanto este es un tema crucial que impacta a las actividades de valor. 


\section{Estefany Robles}

En razón de lo planteado, es importante la actualización permanente de los planes de higiene y seguridad industrial en el trabajo, producto que éstos inciden de manera directa en la producción. Más sin embargo en lo referido al tema de salud laboral no sólo debe verse desde la perspectiva económica, sino que la empresa debe velar por que los trabajadores tengan las herramientas necesarias que le permitan realizar sus labores con el menor riesgo posible, éste debería ser la razón fundamental de la implementación de dichos planes.

\section{Relaciones Laborales}

Para, Cabello (2008) las relaciones laborales son los mecanismos utilizados por la empresa para generar una cultura organizacional en la que los empleados se sientan motivados a participar en los procesos de decisión, a ser creativos y tener iniciativa, enlazadas a la razón de ser de la compañía.

Mientras que Chiavenato (2009) denomina las relaciones laborales de la misma forma, relacionándolas con el trabajo del personal incluyendo las negociaciones y acordadas con los sindicatos.

Por su parte, Robbins (2006) expresa que uno de los aspectos fundamentales de las relaciones obrero patronales lo constituye el momento en el que ambas partes se sientan alrededor de la mesa a negociar. De hecho, algunas leyes laborales ordenan que así sea, y les exigen a ambas partes los sindicatos y patrones que realicen negociaciones de buena fe. Esto exige que ambas partes estén dispuestas a llegar a un acuerdo. Es decir, su esfuerzo debe tener un impacto positivo sobre el proceso, para alcanzar la meta última que consiste en llegar a un acuerdo.

De la misma manera, Mondy (2006) afirma que relaciones laborales juegan un papel importante dentro del desarrollo de la administración de los recursos humanos, las cataloga como un punto álgido dentro de la organización mostrando el concepto de sindicatos los cuales fungen como mediadores en la discusión entre los patrones y los empleados, buscando en primera línea, ser garantes del cumplimiento de las responsabilidades de la empresa para con su capital humano.

En razón a los planteamientos de los autores, las relaciones laborales van a jugar un papel fundamental dentro de una organización, por cuanto en la medida que tenga conocimiento de los compromisos a cumplir, así podrá exigir los derechos que le 


\section{Estefany Robles}

corresponden como trabajador.

\section{Desarrollo Personal}

Según Chiavenato (2009) los procesos de desarrollo de personas incluyen las actividades de entrenamiento, desarrollo de personal y desarrollo organizacional, estas representan las inversiones realizadas en el personal. Así pues, estas se destacan por ser el único elemento vivo e inteligente de la organización.

Al respecto Robbins (2006) afirma que el desarrollo del empleado, se orienta hacia el futuro y tiene se enfoca más con la educación que con la capacitación del empleado en un puesto de trabajo en específico, entendiendo que todos independientemente de su nivel pueden desarrollarse. Mariscan (2008), relaciona el desarrollo de las personas con su carrera específica y las técnicas que la integran, pero también con los requerimientos culturales de la empresa, así mismo su entrenamiento depende del cargo que este va a desempeñar.

En este sentido, el desarrollo de personal está integrado entre el equilibrio del trabajo tomando en cuenta la vida personal obteniendo como resultado un componente importante para la satisfacción y lealtad por parte de los trabajados, es decir, las empresas que se preocupen por el equilibrio emocional de sus trabajadores serán aquellas que pueden lograr empleados eficientes y satisfechos, permitiendo así que los mismos estén en constante actualización y formación de su profesión. Las personas o instituciones que este en desacuerdo con estas políticas deben ser frenadas y por ende penalizadas.

\section{Gestión del Impacto Ambiental}

Autores como Gómez (2013), afirma que la gestión ambiental opera sobre el concepto de impacto ambiental y su diagnóstico. El término impacto se refiere a la alteración con respecto a las actividades humanas realizadas las cuales se introducen en el medio ambiente, aunque el calificativo ambiental alude a la interpretación de tales alteraciones en términos de salud y bienestar humano. EI autor refiere tres tipos de impactos ambientales entre los cuales se cuentan los positivos y los negativos, reversibles e irreversibles, directos o inducidos, permanentes o temporales, simples $o$ acumulativos, sinérgicos o no. En este orden 


\section{Estefany Robles}

de ideas continúa su planteamiento expresando que la gestión puede intervenir por vía preventiva, correctora o curativa.

De igual forma, Vega (2005) afirma que la gestión del impacto ambiental debe dirigirse hacia la sostenibilidad ambiental del desarrollo y deben estar diseñados de manera tal que la empresa logre enfocarse estratégicamente en los programas emitidos por el gobierno nacional y por medio de los cuales debe direccionar sus prácticas en materia ambiental.

En el mismo orden de ideas, para Pousa, (2007) es un instrumento o herramienta que posee la empresa como ayuda en las actividades de su gestión medioambiental. Aporta la base para orientar, encauzar, medir y evaluar en funcionamiento de la empresa con el fin de asegurar que sus operaciones se llevan a cabo de manera consecuente con la reglamentación aplicable y con la política corporativa en dicho sentido.

\section{METODOLOGÍA}

El paradigma o tradición de la presente investigación es cualitativa, a saber de Pérez Serrano (2008) es considerada como un proceso activo, sistemático y riguroso de indagación dirigida, en la que se toman decisiones sobre lo investigable en tanto se está en el campo de estudio. Igualmente se centra en el estudio de los significados de las acciones humanas y de la vida social. Cabe agregar que, la investigación cualitativa en el área de estudio, puede referir lo dicho por Paz (2013), en cuanto que es una actividad sistemática orientada a la comprensión en profundidad de fenómenos sociales, a la transformación de prácticas y escenarios sociales, a la toma de decisiones y también hacia el descubrimiento y desarrollo de un cuerpo organizado de conocimientos.

Del mismo modo, es importante destacar que cada paradigma tiene una manera de ver la realidad - el hombre, la sociedad, que se corresponde con lo ontológico, una concepción de lo que es conocimiento, cómo se construye, lo epistemológico, una manera de ver el papel de los valores en dicho proceso, lo axiológico y la forma de abordar el proceso de la investigación, que lo constituye la metodología. Desde el paradigma cualitativo, cada una de estas dimensiones se orienta el proceso a considerar en este tipo de investigación. 


\section{Estefany Robles}

En cuanto a la dimensión ontológica, está referida a la forma y naturaleza de la realidad social y natural, lo que puede y debe ser conocido, una realidad que envuelve el mundo emocional de los actores participantes en el estudio $y$, que puede ser socialmente interpretada, comprendida y conceptualizada a través de un lenguaje de matiz profunda.

Cuya aceptación permite como investigador abordar a un sujeto que dentro de su contexto o medio de relaciones personales siente y expresa con mayor fuerza sus sentimientos, producto de la confianza y seguridad, valorando en todo momento su punto de vista, interpretaciones y significaciones, tomando como referente su lenguaje oral y corporal, ya como expresa Can y Kemmis (1986), el carácter social de las acciones implica que estas surgen de los retos de los significados conferidos a los individuos.

Por lo tanto, la especificación de los conocimientos y la naturaleza del fenómeno social que se aborda en el estudio e interpretación de los estados emocionales, es comprendida a través de la realidad vivida por los actores, es decir las opiniones, manifiestos están representado por intencionalidades, significados y motivaciones, estados de ánimo e interpretaciones del mundo vivido por sus propios protagonistas. Desde la dimensión epistemológica referida a la forma o manera de cómo se construye el conocimiento y cómo se configura la relación investigadora investigado, se asume la ínter subjetividad entre el mundo que subyace desde las vivencias del investigador, formando parte de la realidad investigada y los actores sociales, quienes a través de sus testimonios versionan esa realidad vivida.

Pues, se trata de comprender la investigación como un proceso dialógico en el que investigador y actores sociales participantes del estudio se comunican en los diferentes espacios sociales en que vive. Donde la información que producen éstos actores por diferentes caminos, viene a constituir el material privilegiado para la construcción y reconstrucción de conocimientos, en el curso en el que el investigador confronte sus pensamientos con la infinidad de eventos empíricos que coexisten en el proceso investigativo.

Por otro lado, se abordó la dimensión metodológica que permitió indagar e interpretar las significaciones humanas, es decir, comprender los significados que los actores sociales vienen aportando desde el interactuar con sus compañeros, así 


\section{Estefany Robles}

como el manejo y uso de las emociones desde la gerencia educativa. De allí que ésta dimensión sitúa las diferentes vías o formas de investigación que emergen de manera natural y se construyen en la medida que se avanza en el proceso de la misma, a través de la cual se pueden recabar distintas versiones y perspectivas de la comunidad en estudio.

Se considera entonces, en la investigación cualitativa no existe un método único de investigación; al contrario, existen múltiples alternativas metodológicas para conocer lo social, el fenómeno humano y los procesos que ocurren en la sociedad.

Por esta razón, la realidad puede ser abordada desde los diferentes métodos de la investigación, siendo uno de ellos el fenomenológico, el cual comprende las realidades cuya naturaleza y estructura depende de las personas que las viven y experimentan, permitiendo la comprensión del fenómeno de estudio.

Según Martínez (2009), lo define como el estudio de los fenómenos tal cual son experimentados, vividos y percibidos por el hombre cuya naturaleza y estructura peculiar solo pueden ser captadas desde el marco de referencia del sujeto que las vive y experimenta; es un método individual, con resultados válidos en determinado tiempo y espacio, y para el sujeto que experimenta las vivencias.; método que orientará el estudio.

\section{CONCLUSIONES}

Al analizar la Responsabilidad social empresarial hay aspectos de interés que deben ser tomados en cuenta entre los cuales cabe mencionar: que la misma no debe verse como una simple acción complementaria y oportunista de una empresa para lograr beneficios internos y externos a fin de crear una imagen ante la sociedad, por el contrario la RSE debe ser reconocida como un nuevo paradigma que exige gestión estratégica que genera ventaja competitiva y que como tal, debe ser aprovechada para la permanencia de una empresa en un mercado tan cambiante.

Otro elemento fundamental es que la RSE no es una práctica aislada e individual, debe ser integral, surge como compromiso de todos y debe incluir toda la estructura organizativa de la organización. Es decir, los integrantes de la empresa deben incluirla en sus planificaciones diarias, de allí entonces la razón fundamental de las estrategias a emplear, las cuales deben ir en la búsqueda de mecanismos que 


\section{Estefany Robles}

activen ese liderazgo estratégico con visión social prospectiva enmarcados siempre en la búsqueda de la calidad y el éxito organizacional.

La RSE debe ir siempre en la búsqueda de programas y políticas que brinden seguridad y confianza para lograr los objetivos de la empresa, es decir no debe estar sujeta a respuestas momentáneas. Por lo tanto, debe incluir la generación de valor agregado, que considere la mejoría en el bienestar y calidad de vida de la sociedad. La gestión de la RSE debe generar ventajas competitivas, iniciando un proceso en que se integren prácticas responsables en su misión y visión. Las mismas deben estar enmarcadas en la diferenciación, como estrategia prioritaria de la empresa, buscando con ello apalancarse en el entorno internacional convulsionado y competitivo, para llegar a convertir la RSE en una alternativa eficiente y eficaz dentro de la organización. De tal manera que, para ser participativo en este ámbito, se necesita respetar el medio ambiente y el sistema ecológico.

Es importante mencionar al benchmarking como herramienta gerencial de divulgación y que coadyuve a elevar la imagen corporativa de las organizaciones, esto permitirá que la responsabilidad social no sólo se aplique internamente, sino que puede hacerse extensiva al ámbito internacional, valiéndose para ello de gestores con personal calificado, actualizado y formado de manera permanente para poder posicionarse en dichos mercados.

\section{REFERENCIAS CONSULTADAS}

1. Álvarez-Meneses, T. (2013). La planificación turística: un aspecto clave para el desarrollo sostenible y regional de Boyacá. Revista de Investigación, Desarrollo e Innovación, 3 (2), 101-110. doi: http://dx.doi.org/10.19053/20278306.2169.

2. Aldana, J., \& Piña, J. (2017). Calidad del servicio prestado al cliente por los instructores de gimnasios. Revista Arbitrada Interdisciplinaria Koinonía, 2(3), 172-197. Recuperado de http://fundacionkoinonia.com.ve/ojs/index.php/revistakoinonia/article/view/59/4 6

3. Arias, E., \& Sarmiento, D. (2016). Responsabilidad social empresarial en Falcón: Un análisis de contenido. Revista Arbitrada Interdisciplinaria Koinonía, 1(1), 22-42. Recuperado de http://fundacionkoinonia.com.ve/ojs/index.php/revistakoinonia/article/view/13/6 


\section{Estefany Robles}

4. Arnal, Capriotti (2010) Responsabilidad Social Empresarial - Bidireccional. (Libro en Línea). Disponible: www.bidireccional.net/Blog/Libro_RSE.pdf (Consulta 2019 junio 29).

5. Balza-Franco, V., \& Cardona-Arbeláez, D. (2015). La responsabilidad social empresarial y la lucha contra la pobreza. Saber, Ciencia y Libertad, 10 (1), 115-124. doi: http://dx.doi.org/10.22525/sabcliber. 2015v10n1.115124.

6. Becerra-Gualdrón, C. J., \& Cruz-Vásquez, J. L. (2014). Diagnóstico de la competitividad agroindustrial en el departamento de Boyacá para el año 2011. Revista de Investigación, Desarrollo e Innovación, 4 (2), 111-123. Doi: http://dx.doi.org/10.19053/20278306.2961

7. Bericat, E. y Echavarren, J. (2008). Andalucía 2020: Escenarios Previsibles. España. Fundación Centro de Estudios Andaluces. (Libro en Línea). Disponible: https://books.google.co.ve (Consulta 2019 junio 19).

8. Carneiro-Caneda, M. (2013). La responsabilidad social corporativa interna: la nueva frontera de los recursos humanos. Aedipe: Revista de la Asociación Española de Dirección de Personal, 27, 42-48.

9. Carr, W. y Kemmis, S. (1986). Teoría crítica de la enseñanza. La investigación acción en la formación del profesorado. Barcelona: Martínez Roca.

10. Chiavenato. I. (2009). Administración de Recursos Humanos. 5ta Edición Colombia. (Libro en Línea). Disponible: https://books.google.co.ve (Consulta 2019 junio13).

11. Comisión de las Comunidades Europeas, CCE. (2011). El libro verde: Fomentar un marco europeo para la responsabilidad social de las empresas, 1-35.

12. Cortés, J. (2012). Seguridad e Higiene en el Trabajo $3^{a}$. Edición Editorial Alfa Omega. México (Libro en Línea). Disponible: https://books.google.co.ve (Consulta 2019 junio19).

13. Delgado, S. (2012) Recursos Humanos y Responsabilidad Social Corporativa. Editorial Publicaciones de la Universidad de Deusto. España. (Libro en Línea) Disponible en http://cv.uoc.edu (Consulta 2019 junio12).

14. Fernández, R. (2009) Responsabilidad Social Corporativa, una Nueva Cultura Empresarial. Editorial Melvin, C.A. España (Libro en Línea). Disponible: https://books.google.co.ve (Consulta 2019 julio 02).

15. García-Rubiano, M., \& Forero-Aponte, C. (2015). Contrato psicológico y cambio organizacional en una entidad perteneciente al sector terciario de la ciudad de Bogotá, Colombia. Revista de Investigación. Desarrollo e Innovación, 6 (1), 15-28. doi: http://doi.org/10.19053/20278306.4047 
16. Grupo Gerdau (2013) Mecanismos para que los accionistas y empleados provean recomendaciones o indicaciones al cuerpo superior de gobierno. http://www.gerdau.cl/Reporte_RSE_2013/indice-gri.php. (Consulta 2019 junio 29).

17. Guédez, V. (2006). Ética y Práctica de la Responsabilidad Social Empresarial: el Aporte de la Empresa al Capital Social. Venezuela. Editorial Planeta Venezolano. (Libro en Línea). Disponible: https://books.google.co.ve (Consulta 2019 junio 13).

18. Güerere, J. (2008). Empresa Responsable, Empresa Rentable: Gestión de un Negocio Lucrativo y Socialmente Responsable. Venezuela. Impresos Grafifor, C.A (Libro en Línea). Disponible: https://books.google.co.ve (Consulta 2019 junio 13).

19. Guibert, J. (2009) Responsabilidad Social Empresarial. Universidad de Deusto. San Sebastián. (Libro en Línea). Disponible: https://books.google.co.ve (Consulta 2019 junio 02).

20. Libro Verde comisión de las Comunidades Europeas (2011) Consultado el 29 junio de 2019 en: www.jussemper.org/Inicio/.../libroverdedelaue.html - Madrid España.

21. López, M. y Grandio, A. (2010). Capital Humano como Fuente de ventajas competitivas: algunas Reflexiones y experiencias. España. Editorial Gestiblo, S.L. (Libro en Línea). Disponible: https://books.google.co.ve (Consulta 2019 julio 13).

22. Marchart, M. (2006) La Responsabilidad Social Corporativa Interna. (Libro en Línea). Disponible: https://books.google.co.ve (Consulta 2019 junio 08).

23.21. Martínez, M. (2009). Ciencia y arte en la metodología cualitativa. Editorial Trillas. México.

24. Martínez-Bernal, M. S. (2013). Determinación de la productividad y competitividad de la pequeña minería del distrito minero del norte de Boyacá. Revista de Investigación, Desarrollo e Innovación, 3 (2), 72-86. doi: $10.19053 / 20278306.2168$

25. McWilliams, A., \& Siegel, D. (2011). Corporate social responsibility: A theory of the firm perspective. Academy of management Review, 26 (1), 117-127. doi: http://doi.org/10.5465/AMR.2001.4011987.

26. Molto, G. (2013) La imputación de Responsabilidades y el Marco Jurídico de la Prevención de Riesgos Laborales. Mc Grau Hill. Madrid. (Libro en Línea). Disponible: https://books.google.co.ve (Consulta 2019 julio 13). 


\section{Estefany Robles}

27. Moreno, A., Uriarte, L. M., \& Topa, G. (2010). La responsabilidad social empresarial: Oportunidades estratégicas, organizativas y de recursos humanos. Ediciones Pirámide.

28. Parra-Penagos, C., \& Rodríguez-Fonseca, F. (2016). La capacitación y su efecto en la calidad dentro de las organizaciones. Revista de Investigación, Desarrollo e Innovación, 6 (2), 131-146. doi: http://dx.doi.org/10.19053/20278306.4602.

29.Paz, S (2013) "Investigación Cualitativa en Educación. Fundamentos y Tradiciones". Madrid. Mc Graw and Hill Interamericana de España (pp.258).

30. Pérez Serrano, G. (2008). Investigación cualitativa: Retos e Interrogantes. La Investigación-Acción. Tomo I. Madrid: Muralla.

31.Pousa, X. (2007) La gestión Medioambiental: Un Objetivo Común. Cómo Reducir el Impacto Medioambiental de las Actividades. Impreso en España, Ideas propias Editorial. (Libro en Línea). Disponible: https://books.google.co.ve (Consulta 2019 junio 08).

32. Reyno, L. (2007) Responsabilidad Social de la Empresa, volumen 146 (libro en línea). Disponible: https://books.google.co.ve (consulta 2019 junio 12).

33. Ríos, P. (2018). Metodología de la Investigación. Un Enfoque Pedagógico. Editorial Cognitus.

34. Robbins, S. (2006). Comportamiento Organizacional. Prentice Hall. México. (Libro en Línea). Disponible: https://books.google.co.ve (Consulta 2019 junio 13).

35. Vega, J. (2005). Responsabilidad Social y los Principios del Desarrollo Sostenible como Fundamentos Teóricos de la Información Socia de la Empresa. Editorial ESIC Madrid España. (Libro en Línea). Disponible: https://books.google.co.ve (Consulta 2019 junio 08).

36. Ventura, F. (2013) responsabilidad social empresarial; dimensión interna y externa. (Libro en Línea). 3 era Edición Disponible: http//www.cedro.org (Consulta 2019 junio 19).

37. Vergara-Arrieta, J. J. (2015). Los sistemas de medición del desempeño estratégico frente a la competitividad y sustentabilidad. Saber, Ciencia y Libertad, $10 \quad$ (2), $1726 . \quad$ Recuperado de: https:// dialnet.unirioja.es/servlet/articulo?codigo $=5295019$ 


\section{Estefany Robles}

\section{REFERENCES CONSULTED}

1. Álvarez-Meneses, T. (2013). Tourism planning: a key aspect for the sustainable and regional development of Boyacá. Journal of Research, Development and Innovation, 3 (2), 101-110. doi: http://dx.doi.org/10.19053/20278306.2169.

2. Aldana, J., \& Piña, J. (2017). Quality of service provided to the client by gym instructors. Interdisciplinary Arbitrated Review Koinonía, 2 (3), 172-197. Recovered from http://fundacionkoinonia.com.ve/ojs/index.php/revistakoinonia/article/view/59/4 6

3. Arias, E., \& Sarmiento, D. (2016). Corporate social responsibility in Falcón: A content analysis. Interdisciplinary Arbitrated Review Koinonía, 1 (1), 22-42. Recovered from http://fundacionkoinonia.com.ve/ojs/index.php/revistakoinonia/article/view/13/6

4. Arnal, Capriotti (2010) Corporate Social Responsibility - Bidirectional. (Book online). Available: www.bidirecional.net/Blog/Libro_RSE.pdf (Check 2019 June 29).

5. Balza-Franco, V., \& Cardona-Arbeláez, D. (2015). Corporate social responsibility and the fight against poverty. Knowledge, Science and Freedom, 10 (1), 115-124. doi: http://dx.doi.org/10.22525/sabcliber. 2015v10n1.115124.

6. Becerra-Gualdrón, C. J., \& Cruz-Vásquez, J. L. (2014). Diagnosis of agroindustrial competitiveness in the department of Boyacá for the year 2011. Journal of Research, Development and Innovation, 4 (2), 111-123. Doi: http://dx.doi.org/10.19053/20278306.2961

7. Bericat, E. and Echavarren, J. (2008). Andalucía 2020: Predictable Scenarios. Spain. Andalusian Studies Center Foundation. (Book online). Available: https://books.google.co.ve (Check 2019 June 19).

8. Carneiro-Caneda, M. (2013). Internal corporate social responsibility: the new frontier of human resources. Aedipe: Magazine of the Spanish Association of Personnel Management, 27, 42-48.

9. Carr, W. and Kemmis, S. (1986). Critical teaching theory. Action research in teacher training. Barcelona: Martínez Roca.

10. Chiavenato. I. (2009). Human resources management. 5th Edition Colombia. (Book online). Available: https://books.google.co.ve (Check 2019 June13).

11. Commission of the European Communities, CCE. (2011). The green paper: Promote a European framework for corporate social responsibility, 1-35. 


\section{Estefany Robles}

12. Cortés, J. (2012). Occupational Health and Safety 3rd. Editorial Edition Alfa Omega. Mexico (Online Book). Available: https://books.google.co.ve (Check 2019 June19).

13. Delgado, S. (2012) Human Resources and Corporate Social Responsibility. Editorial Publications of the University of Deusto. Spain. (Book Online) Available at http://cv.uoc.edu (Check 2019 June12).

14. Fernández, R. (2009) Corporate Social Responsibility, a New Business Culture. Editorial Melvin, C.A. Spain (Book Online). Available: https://books.google.co.ve (Check 2019 July 02).

15. García-Rubiano, M., \& Forero-Aponte, C. (2015). Psychological contract and organizational change in an entity belonging to the tertiary sector of the city of Bogotá, Colombia. Research Magazine Development and Innovation, 6 (1), 15-28. doi: http://doi.org/10.19053/20278306.4047

16. Gerdau Group (2013) Mechanisms for shareholders and employees to provide recommendations or indications to the upper body of government. http://www.gerdau.cl/Reporte_RSE_2013/indice-gri.php. (Check 2019 June 29).

17. Guédez, V. (2006). Ethics and Practice of Corporate Social Responsibility: the Company's Contribution to Social Capital. Venezuela. Editorial Venezuelan Planet. (Book online). Available: https://books.google.co.ve (Check 2019 June 13).

18. Güerere, J. (2008). Responsible Company, Profitable Company: Management of a Lucrative and Socially Responsible Business. Venezuela. Impresos Grafifor, C.A (Online Book). Available: https://books.google.co.ve (Check 2019 June 13).

19. Guibert, J. (2009) Corporate Social Responsibility. University of Deusto. Saint Sebastian. (Book online). Available: https://books.google.co.ve (Check 2019 June 02).

20. Green Paper commission of the European Communities (2011) Accessed on June 29, 2019 at: www.jussemper.org/lnicio/.../libroverdedelaue.html - Madrid Spain.

21. López, M. and Grandio, A. (2010). Human Capital as a Source of competitive advantages: some Reflections and experiences. Spain. Editorial Gestiblo, S.L. (Book online). Available: https://books.google.co.ve (Check 2019 July 13).

22. Marchart, M. (2006) Internal Corporate Social Responsibility. (Book online). Available: https://books.google.co.ve (Check 2019 June 08). 


\section{Estefany Robles}

23. Martínez, M. (2009). Science and art in the qualitative methodology. Editorial Trillas. Mexico.

24. Martínez-Bernal, M. S. (2013). Determination of productivity and competitiveness of small mining in the mining district of northern Boyacá. Journal of Research, Development and Innovation, 3 (2), 72-86. doi: 10.19053 / 20278306.2168

25. McWilliams, A., \& Siegel, D. (2011). Corporate social responsibility: A theory of the firm perspective. Academy of management Review, 26 (1), 117-127. doi: http://doi.org/10.5465/AMR.2001.4011987.

26. Molto, G. (2013) The imputation of Responsibilities and the Legal Framework of Occupational Risk Prevention. Mc Grau Hill Madrid. (Book online). Available: https://books.google.co.ve (Check 2019 July 13).

27. Moreno, A., Uriarte, L. M., \& Topa, G. (2010). Corporate social responsibility: Strategic, organizational and human resources opportunities. Pyramid editions.

28. Parra-Penagos, C., \& Rodríguez-Fonseca, F. (2016). Training and its effect on quality within organizations. Journal of Research, Development and Innovation, 6 (2), 131-146. doi: http://dx.doi.org/10.19053/20278306.4602.

29.Paz, S (2013) "Qualitative Research in Education. Foundations and Traditions". Madrid. Mc Graw and Hill Interamerican of Spain (pp. 258).

30. Pérez Serrano, G. (2008). Qualitative research: Challenges and Questions. Action Research. Volume I. Madrid: Wall.

31.Pousa, X. (2007) Environmental Management: A Common Objective. How to Reduce the Environmental Impact of Activities. Printed in Spain, Own ideas Editorial. (Book online). Available: https://books.google.co.ve (Check 2019 June 08).

32. Reyno, L. (2007) Corporate Social Responsibility, volume 146 (online book). Available: https://books.google.co.ve (see 2019 June 12).

33. Ríos, P. (2018). Investigation methodology. A Pedagogical Approach. Cognitus editorial.

34. Robbins, S. (2006). Organizational behavior. Prentice Hall. Mexico. (Book online). Available: https://books.google.co.ve (Check 2019 June 13).

35. Vega, J. (2005). Social Responsibility and the Principles of Sustainable Development as Theoretical Foundations of the Company's Social Information. Editorial ESIC Madrid Spain. (Book online). Available: https://books.google.co.ve (Check 2019 June 08). 
Revista Interdisciplinaria de Humanidades, Educación, Ciencia y Tecnología

Año V. Vol. V. N09. Julio - Diciembre 2019

Hecho el depósito de ley: pp201602FA4721

ISSN-L: 2542-3029; ISSN: 2610-802X

Universidad Nacional Experimental Francisco de Miranda (UNEFM). Santa Ana de Coro. Venezuela

Estefany Robles

36. Ventura, F. (2013) corporate social responsibility; internal and external dimension. (Book online). 3rd Edition Available: http // www.cedro.org (Check 2019 June 19).

37. Vergara-Arrieta, J. J. (2015). The measurement systems of strategic performance against competitiveness and sustainability. Saber, Ciencia y Libertad, 10 (2), 1726. Retrieved from: https: // dialnet.unirioja.es/servlet/articulo?codigo $=5295019$

C2019 por los autores. Este artículo es de acceso abierto y distribuido según los términos y condiciones de la licencia Creative Commons Atribución-NoComercial-Compartirlgual 4.0 Internacional (CC BY-NC-SA 4.0) (https://creativecommons.org/licenses/by-nc-sa/4.0/). 